\title{
Probing the sea of galactic cosmic rays with Fermi-LAT
}

\author{
Felix Aharonian* \\ Dublin Institute for Advanced Studies, 31 Fitzwilliam Place, Dublin 2, Ireland, \\ and Max-Planck-Institut für Kernphysik, P.O. Box 103980, 69029 Heidelberg, Germany \\ Giada Peron $\oplus^{\dagger}$ \\ Max-Planck-Institut für Kernphysik, P.O. Box 103980, 69029 Heidelberg, Germany \\ Ruizhi Yang ${ }^{\ddagger}$ \\ Max-Planck-Institut für Kernphysik, P.O. Box 103980, 69029 Heidelberg, Germany \\ and Department of Astronomy, School of Physical Sciences, \\ University of Science and Technology of China, Hefei, Anhui 230026, China \\ Sabrina Casanova $\oplus^{\|}$ \\ Max-Planck-Institut für Kernphysik, P.O. Box 103980, 69029 Heidelberg, Germany, \\ and Institute of Nuclear Physics, Radzikowskiego 152, 31-342 Krakow, Poland \\ Roberta Zanin ${ }^{\S}$ \\ Max-Planck-Institut für Kernphysik, P.O. Box 103980, 69029 Heidelberg, Germany
}

(Received 3 December 2018; revised manuscript received 24 July 2019; accepted 5 March 2020; published 15 April 2020)

High energy $\gamma$-rays from giant molecular clouds (GMCs) carry direct information about the spatial and energy distributions of galactic cosmic rays (CRs). The recently released catalogs of GMCs contain sufficiently massive clouds to be used as barometers for probing, through their $\gamma$-ray emission, the density of CRs throughout the galactic disk. Based on the data of Fermi-LAT, we report the discovery of $\gamma$-ray signals from nineteen GMCs located at distances up to $12.5 \mathrm{kpc}$. The galactocentric radial distribution of the $\mathrm{CR}$ density derived from the $\gamma$-ray and $\mathrm{CO}$ observations of these objects, as well as from some nearby clouds that belong to the Gould Belt complex, unveil a homogeneous "sea" of CRs with a constant density and spectral shape close to the flux of directly (locally) measured CRs. This concerns the galactocentric distances exceeding $8 \mathrm{kpc}$, as well as the Sagittarius B complex, in the region of the Galactic Center. On the other hand, for the galactocentric distances between 4 and $8 \mathrm{kpc}$, we found noticeable deviations from the CR sea level; in some locations, GMCs are characterized by enhanced CR density. This could be the result of a possible global increase of the level of the CR sea towards the Galactic Center and/or by the presence of recent CR accelerators close to some specific clouds.

DOI: $10.1103 /$ PhysRevD.101.083018

\section{INTRODUCTION}

The recent years have been marked by impressive progress in the precision and quality of direct cosmic ray

\footnotetext{
*felix.aharonian@mpi-hd.mpg.de

†peron@mpi-hd.mpg.de

*yangrz@ustc.edu.cn

roberta.zanin@mpi-hd.mpg.de

sabrinacasanova@gmail.com
}

Published by the American Physical Society under the terms of the Creative Commons Attribution 4.0 International license. Further distribution of this work must maintain attribution to the author(s) and the published article's title, journal citation, and DOI. Open access publication funded by the Max Planck Society.
(CR) measurements. Yet, the key issues concerning the origin of galactic CRs are not fully understood and resolved.

A breakthrough in the field is expected from $\gamma$-ray observations. This concerns both the acceleration and propagation aspects of $\mathrm{CR}$ studies. While the detection and identification of $\gamma$-ray sources unveil the sites of CR production, the diffuse $\gamma$-ray emission of the galactic disk (GD) contains information about the spatial and energy distributions of CRs in the Milky Way. The accumulation and effective mixture of relativistic particles through their convection and diffusion in the interstellar magnetic fields results in the formation of the so-called sea of galactic CRs. The level and the energy spectrum of the CR sea is determined by the operation of all galactic accelerators over the confinement time of CRs. At low $(\mathrm{GeV})$ energies it 
is estimated about $10^{7}$ years, and decreases with energy as $E^{-\delta}$, with $\delta \sim 0.3-0.5$. The "lifetimes" of potential galactic $\mathrm{CR}$ factories typically are shorter than the CR confinement time in the GD. Therefore one should expect a smooth and rather homogeneous distribution of CRs on large $(\mathrm{kpc})$ scales. The homogeneity of CR distribution can be violated on smaller scales, in particular in the proximity of recent or currently operating particle accelerators.

In the GD, the diffuse galactic $\gamma$ radiation is produced by relativistic electrons, protons and nuclei interacting with the interstellar gas and radiation fields. The energy interval from $100 \mathrm{MeV}$ to $100 \mathrm{GeV}$ is dominated by $\gamma$ rays from the decays of secondary $\pi^{0}$ mesons [1-3]. Thus, the diffuse $\gamma$-ray emission contains essential information about the gas density-weighted mean density of galactic CRs: $\rho_{\mathrm{CR}} \propto$ $F_{\gamma} / N_{\mathrm{H}}$, where $F_{\gamma}$ and $N_{\mathrm{H}}$ are the $\gamma$-ray flux and the gas column density in the given direction of ISM, respectively. Under certain assumptions, this information can be used to derive the density of CRs as a function of distances from the Galactic Center [4-6]. The method has certain limitations. First of all, the measured CR density is the mean value averaged over the vast areas of the galactocentric rings (typically, $\gtrsim 10 \mathrm{kpc}^{2}$ ). Therefore it can provide only integral information about the CR density. Second, the method assumes cylindrical symmetry, which perhaps could be considered a reasonable approximation but yet remains an ad hoc assumption. In particular, it is not apparent that the relevant parameters like the CR diffusion coefficient, the spatial distribution of CR accelerators, and hence ultimately the CR density, do not vary on very large (multi-kpc) scales within the galactocentric rings. Moreover, the measurement of the CR density through the diffuse gamma-ray emission is affected by the contamination from the diffuse inverse Compton emission, and unresolved $\gamma$-ray sources. Finally, the gas column density is dominated by the contribution of a limited number of giant molecular clouds (GMCs), thus the derived CR density corresponds to the value averaged over specific locations occupied by these clouds.

In this regard, $\gamma$ rays from individual GMCs can provide more straightforward and differential (localized in space) information about CRs. Moreover, the high gas density of GMCs and their compactness make negligible the contribution from the inverse Compton component of diffuse background radiation and significantly reduces the level of potential contamination from other large-scale sources. Thus, GMCs can be treated as unique CR barometers distributed throughout the Galaxy $[7,8]$. The case of passive GMCs, i.e., clouds located far from active CR accelerators, is of particular interest. The detection of $\gamma$ rays from such clouds tells us about the level of the CR sea without substantial contamination by particles injected by nearby objects.

The $\gamma$-ray emissivity of a GMC depends on the ratio of timescales of proton-proton interactions and $\mathrm{CR}$ propagation in the cloud. The proposed method of probing the CR density with $\gamma$ rays can be realized provided that CRs freely penetrate the clouds. Although the latter condition cannot be a priori satisfied (see, e.g., Ref. [9]), in the case of "passive" clouds embedded in the CR sea, we can safely assume free CR penetration unless the propagation inside the clouds is dramatically slower compared to the diffusion in the ISM. For the typical parameters of GMCs, the timescales of propagation of CRs through the clouds, assuming that the diffusion coefficient inside the cloud is similar to the one in the ISM [10], does not exceed $10^{4} \mathrm{yrs}$ even for low-energy $(\leq 10 \mathrm{GeV})$ particles. This is shorter, by 2 orders magnitude, than the confinement time of CRs in the Galaxy $\left(10^{6}-10^{7}\right.$ years), as well as the characteristic $p p$ interaction time inside the clouds $\left(\tau_{p p} \simeq 3 \times 10^{5}\left(\mathrm{n} / 10^{2} \mathrm{~cm}^{-3}\right)^{-1} \mathrm{yr}\right)$. Otherwise, for $\gamma$ rays, as products of these interactions, we should expect significantly harder spectra compared to the spectrum of parent protons [10]. While this seems quite unlikely to happen in passive clouds, the impact of $\mathrm{CR}$ propagation effects could be stronger in the case of clouds located in the vicinity of $\mathrm{CR}$ accelerators with the operation timescales much shorter than the CR confinement time in the Galaxy. Therefore, the detection of hard $\gamma$-ray spectra from individual clouds can serve as an indicator of presence of nearby recent or currently active accelerators, and thus should be excluded from the sample of objects to be used for extraction of the CR sea. Apparently, this cannot be done in the case of derivation of the CR sea from the diffuse $\gamma$-ray background, the latter being the superposition of contributions from both "active" and passive clouds, as well as the intercloud regions.

Under the assumption that CRs freely penetrate the cloud, the flux of $\gamma$ rays from a passive cloud depends on a single parameter, the ratio $M / d^{2}$, where $M$ is the cloud mass and $d$ is the distance to the source:

$$
F_{\gamma}\left(E_{\gamma}\right)=\frac{M}{d^{2}} \frac{\xi_{N}}{m_{p}} \int \mathrm{d} E_{p} \frac{\mathrm{d} \sigma}{\mathrm{d} E_{\gamma}} F_{p}\left(E_{p}\right)
$$

Here $m_{p}$ is the proton mass. The parameter $\xi_{N}$ takes into account the contribution of nuclei to the $\gamma$-ray flux; below we will assume $\xi_{N}=1.8$ corresponding to the standard composition of the interstellar medium and CRs [11]. $F_{p}\left(E_{p}\right)$ is the energy distribution of CR protons. The local flux of CRs has been measured with high precision by the AMS collaboration [12]. Above $45 \mathrm{GV}(\sim 45 \mathrm{GeV})$, it is well described, as a function of rigidity, $R$, by the following equation:

$$
F_{p}(R)=C\left(\frac{R}{45 \mathrm{GV}}\right)^{-\gamma}\left[1+\left(\frac{R}{R_{0}}\right)^{\frac{\Delta \gamma}{s}}\right]^{s}
$$


with $\gamma=2.849, R_{0}=336 \mathrm{GV}, \Delta \gamma=0.133$ and $s=0.024$. This presentation accurately describes the recently discovered hardening at $\sim 200 \mathrm{GeV}$. For the differential cross section of $p p$ interactions, $\frac{\mathrm{d} \sigma}{\mathrm{d} E_{\gamma}}$, we use the parametrizations from Ref. [11].

It is convenient [7] to write the ratio $M / d^{2}$ in the normalized form

$$
A=M_{5} / d_{\mathrm{kpc}}^{2},
$$

where $M_{5}=M / 10^{5} M_{\odot}$ and $d_{\mathrm{kpc}}=d / 1 \mathrm{kpc}$. We use it to set the detection threshold of $\gamma$ rays from the clouds by the Fermi-LAT.

In Fig. 1, we show the $\gamma$-ray fluxes calculated for different values of $A$ against the $10-\mathrm{yr}$ sensitivities of Fermi-LAT. The inner sensitivity corresponds to the minimum detectable flux calculated for $l, b=\left(0^{\circ}, 0^{\circ}\right)$; generally, it is valid for the inner part of GD, $|l| \lesssim 60^{\circ}$ and $|b| \lesssim 5^{\circ}$. The outer sensitivity corresponds to the minimum detectable flux of a source located in the region $l, b=\left(0^{\circ}, 30^{\circ}\right)$. But the latter characterizes, in fact, the sensitivity for a significantly broader fraction of the GD: $|l|>60^{\circ}, 5<|b|<45^{\circ}$ ). For details, see the Fermi-LAT performances website. ${ }^{1}$ The curves in Fig. 1 are calculated for different angular extensions of the source $\theta$ by multiplying the sensitivity for the pointlike source by the factor $\sqrt{\sigma_{P S F}^{2}+\theta^{2}} / \sigma_{P S F}$. From Fig. 1 one can see that in the case of sources with angular extensions smaller than $1^{\circ}$, FermiLAT is capable to detect molecular clouds with $A \gtrsim 0.4$. In the case of location of compact clouds in uncrowded regions, the detection threshold can be as small as $A \simeq 0.2$. On the other hand, for very close clouds $(d<1 \mathrm{kpc})$, the parameter $A$ should significantly exceed 1 to compensate the loss of the sensitivity of Fermi-LAT due to the large (several degrees) extensions of clouds. In the opposite case of gas complexes located in the Galactic Center (GC), the reduction of the flux $\left(\propto d^{-2}\right)$ is compensated by the vast masses, $M \sim 10^{7} M_{\odot}$, and small angular extensions of $\sim 10 \mathrm{arcmin}$. This explains why so far positive $\gamma$-ray signals have been reported only from relatively nearby molecular clouds [13-17] and from the Sgr B complex in the GC [18]. The estimates of the CR density in these two essentially different parts of the Galaxy are important but not sufficient for conclusions regarding the overall distribution of CRs in the Milky Way.

For a comprehensive study of the CR sea, many $\gamma$-ray emitting clouds, broadly distributed over the galactic plane, are needed. Until recently, the practical realization of this goal with Fermi-LAT seemed unrealistic. However, the release of a catalog of galactic GMCs [19] containing clouds with unexpectedly large masses dramatically

\footnotetext{
${ }^{1}$ https://www.slac.stanford.edu/exp/glast/groups/canda/lat_ Performance.htm.
}

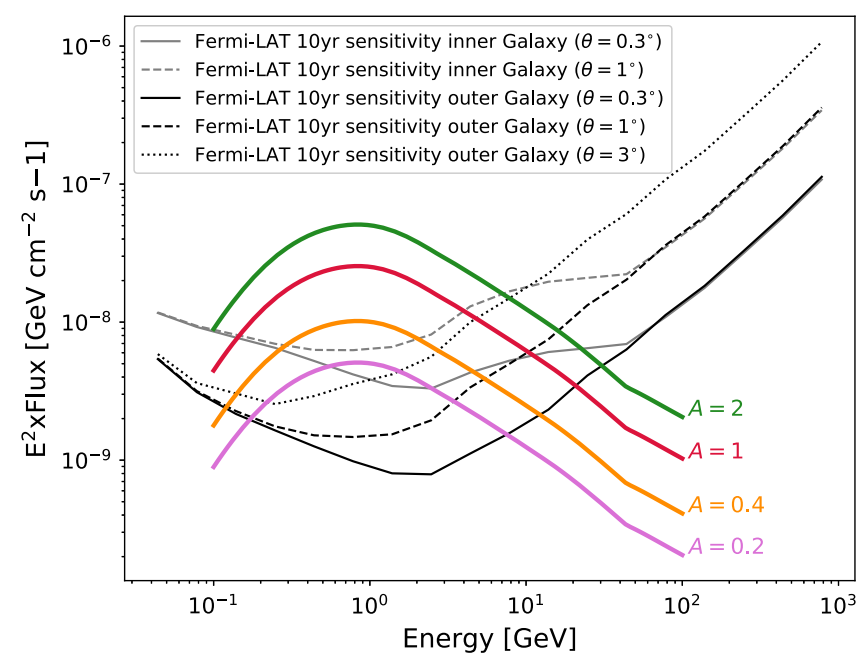

FIG. 1. Gamma-ray fluxes calculated for different values of the A parameter. For comparison, the Fermi-LAT flux sensitivities for different regions of the sky and for different angular extensions of sources are shown.

increased the chances of detection of these clouds in $\gamma$ rays. The above-cited catalog of clouds is based on the $\mathrm{CO}$ line survey conducted by Dame, Hartmann and Thaddeus (DHT) [20], so from now on we will refer to these clouds as DHT clouds. These clouds span almost the entire GD (with the exception of the inner $2 \mathrm{kpc}$ ) and several of them are characterized by sufficiently large values of $A$ to be detected by Fermi-LAT, as shown in Fig. 2.

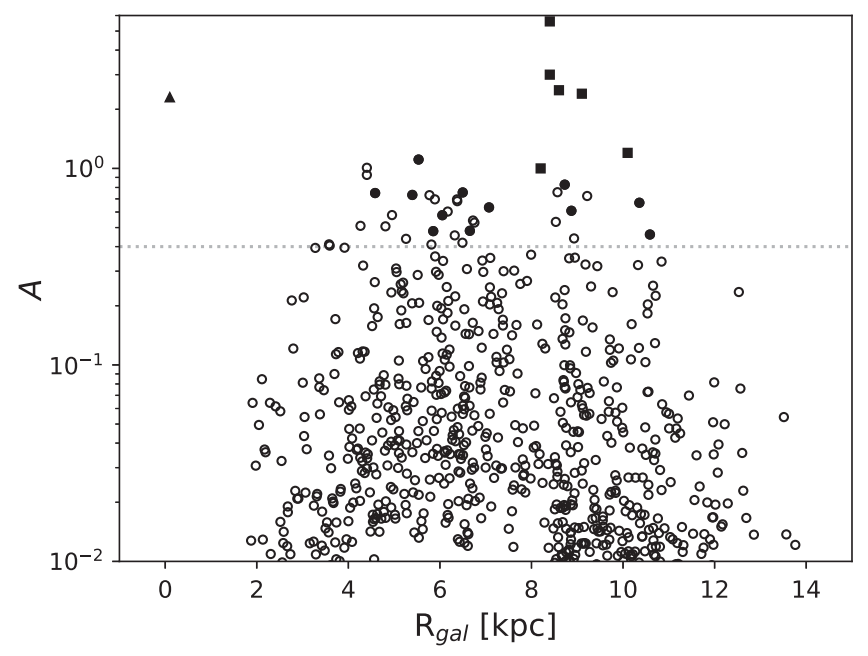

FIG. 2. Distribution of the $A\left(M_{5} / d_{\mathrm{kpc}}^{2}\right)$ parameter of GMCs as a function of the distance from the Galactic Center. The DHT clouds are indicated as circles, the filled symbols indicate the clouds analyzed in this work. We indicate also the parameter for the analyzed nearby molecular clouds (squares) as well as Sagittarius B (triangle). The clouds above the dotted horizontal line have a flux high enough to be detected with the current sensitivity of accumulated Fermi-LAT observations. 
TABLE I. Parameters of the selected GMCs: galactic coordinates $(l, b)$, masses $M$, distances from the Earth $d$, galactocentric distances $R_{\mathrm{GC}}$, and the $A$ parameter. The information about the distant GMCs (the upper part of the table) is derived from the data of Ref. [19]. The distances of nearby clouds (lower part of the table) are from Ref. [21]. The masses of the nearby clouds are calculated from the column densities derived from Planck maps [22]. The mass and the distance to Sgr B are from Refs. [18,23].

\begin{tabular}{|c|c|c|c|c|c|c|}
\hline Cloud & $l(\mathrm{deg})$ & $b(\mathrm{deg})$ & Mass $\left(10^{5} M_{\odot}\right)$ & $\mathrm{d}(\mathrm{kpc})$ & $\mathrm{R}_{\mathrm{GC}}(\mathrm{kpc})$ & $A$ \\
\hline 243 & 42.04 & -0.36 & $30 \pm 10$ & $7.9 \pm 0.6$ & 5.8 & 0.47 \\
\hline 418 & 111.45 & 0.79 & $8 \pm 3$ & $4.1 \pm 0.6$ & 10.6 & 0.46 \\
\hline 429 & 109.84 & -0.29 & $10 \pm 4$ & $3.9 \pm 0.6$ & 10.3 & 0.67 \\
\hline 610 & 142.40 & 1.38 & $0.3 \pm 0.4$ & $0.7 \pm 0.5$ & 8.9 & 0.61 \\
\hline 612 & 126.87 & -0.66 & $0.3 \pm 0.5$ & $0.6 \pm 0.5$ & 8.7 & 0.83 \\
\hline 804 & 328.58 & 0.4 & $24 \pm 8$ & $5.7 \pm 0.6$ & 4.6 & 0.75 \\
\hline 876 & 323.61 & 0.22 & $60 \pm 18$ & $10.2 \pm 0.4$ & 6.0 & 0.58 \\
\hline 877 & 333.46 & -0.31 & $13 \pm 4$ & $3.4 \pm 0.4$ & 5.5 & 1.11 \\
\hline 900 & 318.07 & -0.21 & $47 \pm 14$ & $9.8 \pm 0.4$ & 6.6 & 0.48 \\
\hline 902 & 340.84 & -0.30 & $110 \pm 30$ & $12.5 \pm 0.4$ & 5.4 & 0.73 \\
\hline 933 & 305.49 & 0.11 & $29 \pm 12$ & $6.8 \pm 0.9$ & 7.1 & 0.63 \\
\hline 964 & 345.57 & 0.79 & $3 \pm 2$ & $1.9 \pm 0.6$ & 6.4 & 0.75 \\
\hline Taurus & 171.6 & -15.8 & 0.11 & $0.141 \pm 0.007$ & 8.4 & 5.6 \\
\hline Lupus & 338.9 & 16.5 & 0.04 & $0.189 \pm 0.009$ & 8.2 & 1.0 \\
\hline Orion A & 209.1 & -19.9 & 0.55 & $0.43 \pm 0.02$ & 8.4 & 3.0 \\
\hline Cepheus & 110.7 & 12.6 & 2.13 & $0.92 \pm 0.05$ & 8.6 & 2.5 \\
\hline MonOB1 & 202.1 & 1.0 & 1.33 & $0.75 \pm 0.03$ & 9.1 & 2.4 \\
\hline Maddalena & 216.5 & -2.5 & 5.29 & $2.1 \pm 0.1$ & 10.1 & 1.2 \\
\hline Sgr B & 0.65 & -0.05 & 150 & $7.9 \pm 0.8$ & 0.1 & 2.3 \\
\hline
\end{tabular}

\section{THE TARGET SELECTION}

We analyzed 19 GMCs spread over the Milky Way, from local clouds belonging to the Gould Belt complex to the central molecular zone in the Galactic Center region. All the analyzed clouds are listed in Table I and are shown in Fig. 3 in galactocentric coordinates.

The distances to molecular clouds are determined in different ways. For closest clouds we have the precise measurements provided by the stellar-reddening technique $[21,24]$. For distant objects, we need to rely on the kinematic distance method (see e.g., Ref. [19]), which has a lower accuracy. Note that even though the uncertainties in the distance measurements of clouds are large, they do not impact the extraction of the CR parameters (see Appendix A).

We differentiate the selected objects into Nearby clouds, DHT clouds and Sgr B.

a. Nearby clouds. - In the local $(R \sim 200$ to $-500 \mathrm{pc})$ environment, we selected three representative clouds from the Gould Belt complex. The Fermi-LAT data from two of them, Taurus and Orion A, have been analyzed in previous studies [14,15] allowing us to cross-check our results. The third source, Lupus, has not been studied before. In addition, we selected three other relatively nearby clouds located outside the Gould Belt: Monoceros OB1, Cepheus and Maddalena. Note that recent studies revealed two gaseous structures associated with Cepheus and located at distances $\sim 300$ and $\sim 800 \mathrm{pc}$. In this work, we analyze only the distant one. Because of their proximity, all these
GMCs are characterized by large values of the $A$ parameter. Moreover they lie above the galactic plane by several degrees, where the background is reduced thus the FermiLAT sensitivity becomes better.

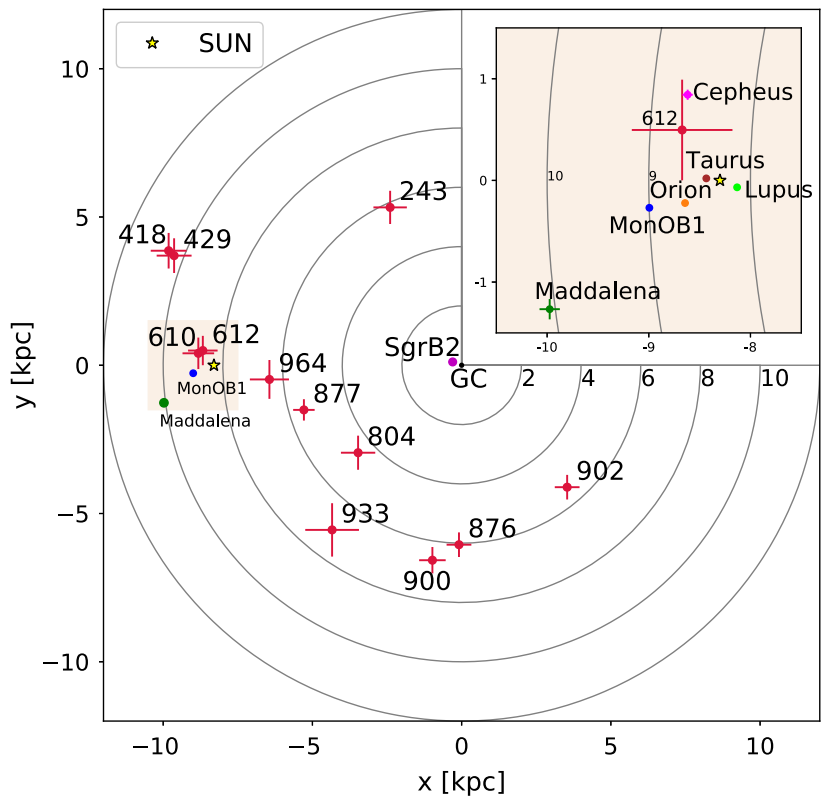

FIG. 3. Positions of the selected GMCs in the galactic plane. In the upper right panel is shown the zoomed region around the Sun with a few selected nearby clouds. The location of the Sgr B complex in the Galactic Center is also shown. The positions and their relative uncertainties are taken from Refs. [19,24,25]. 

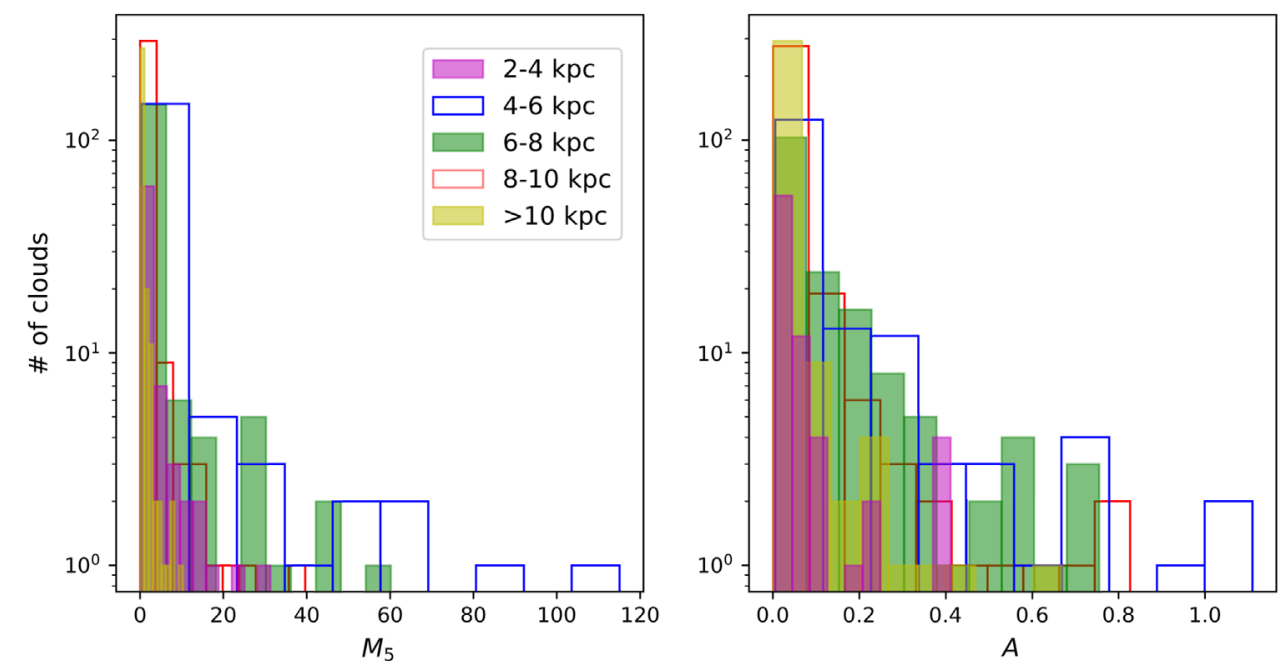

FIG. 4. The histograms show the distributions of mass and A parameter of the DHT clouds [19]. The different colors indicate different galactocentric distance ranges.

b. DHT clouds.-The majority of clouds of our sample consists of clouds from the catalog of [19]. In total, the latter contains 1064 molecular clouds, distributed between the longitudes $180^{\circ}>l>13^{\circ}$ and $348^{\circ}>l>180^{\circ}$, and within the narrow band of latitudes, $-5^{\circ}<b<5^{\circ}$. The range of galactocentric distances of these clouds extends from $\sim 2$ to $\sim 15 \mathrm{kpc}$. Unfortunately the innermost part of the Galaxy, which is of special interest, is not included in Ref. [19].

In Fig. 4 we show the distribution of the clouds in terms of their masses and the $A$ parameter. Approximately $2 / 3$ of them have relatively small masses, $M<10^{5} M_{\odot}$, while a significant fraction, approximately $1 / 4$, has masses between $10^{5}-10^{6} M_{\odot}$. The rest of the clouds, approximately $4 \%$, have masses exceeding $10^{6} M_{\odot}$, including one cloud that is heavier than $10^{7} M_{\odot}$. Note that the most massive clouds are located in the inner Galaxy within 4 and $\sim 6 \mathrm{kpc}$. The large mass and the relatively small angular extensions of these objects, $\lesssim 1^{\circ}$ make them perfect targets for Fermi-LAT.

We selected 12 GMCs from the catalog. The selection was based on the following criteria: (i) the value of the $A$ parameter, (ii) the contribution of the individual clouds to the gas column density along the given line of sight, (iii) the presence of resolved gamma-ray sources in the cloud's proximity.

Remarkably, the very massive GMCs chosen for the analysis appeared to be the dominant objects in terms of contributions to the total column densities in the corresponding directions. The details can be found in the Appendix B. For all clouds, the contributions to the column density of the molecular gas vary between $40 \%$ and $85 \%$; see Table II. This reduces the possible confusion with other background or foreground clouds, thus allowing us to localize the $\gamma$-radiation region with an accuracy comparable to the size of the cloud, typically $10-100 \mathrm{pc}$.
To evaluate the possible confusion with other very bright $\gamma$-ray sources, we used the 3FGL [26] and the HGPS [27] catalogs, and explored the known sources located in the vicinity of our sampled clouds. We discarded the clouds that have many overlapping sources (e.g., clouds 151, 190, 842) and the clouds that are in the proximity of strong H.E.S.S. sources (e.g., 284, 269, 292, 897). The 12 selected clouds do not have identified nearby sources, but some of them overlap with confused sources. In particular, the clouds 610, 902, 933 do have faint nearby background or foreground sources. However, after the fits, they appeared negligible (TS $<10$ ). The clouds 804,877 and 964 have strong (TS $>25$ ) nearby sources. We tested the effect of the existence of these bright sources in the vicinity of the clouds by removing them from the background model. This test showed that while the spectral shapes are

TABLE II. The relative contributions of the selected clouds to the molecular $\left(\mathrm{H}_{2}\right)$ and total $\left(\mathrm{H}_{2}+\mathrm{HI}\right)$ hydrogen column densities towards the directions of these clouds.

\begin{tabular}{lcc}
\hline \hline Cloud & $N_{H}($ cloud $)$ & $\frac{N_{H}(\text { cloud })}{N_{H}\left(H_{2}\right)} \%$ \\
\hline 243 & 53 & 30 \\
418 & 83 & 45 \\
429 & 86 & 31 \\
610 & 82 & 29 \\
612 & 73 & 19 \\
804 & 45 & 26 \\
876 & 53 & 27 \\
877 & 49 & 34 \\
900 & 58 & 32 \\
902 & 47 & 32 \\
933 & 84 & 41 \\
964 & 41 & 28 \\
\hline \hline
\end{tabular}


not affected, the absolute fluxes could vary within $20 \%$, which is smaller than the systematic uncertainties.

c. The Sagittarius B complex.-The dense gas complexes in the Galactic Center region, as parts of the central molecular zone (CMZ), provide an opportunity to probe the CR density at this unique location. The so-called Sgr B complex, which contains the very massive clouds, Sgr B1 and Sgr B2, occupies the region $0.4^{\circ} \leq l \leq 0.9^{\circ}$ and $-0.3^{\circ} \leq b \leq 0.2^{\circ}$. The large distances to these objects, $d_{k p c} \sim 7.9 \pm 0.8$ [23], is compensated by their huge masses. The total mass of this region, derived from infrared observations, results in a quite large value of the $A$ parameter, $A \simeq 2.3$.

\section{DATA ANALYSIS}

We analyzed nine years of Fermi-LAT data, from MET 239557417 (August 4, 2008) to MET 533045411 (November 22, 2017), using the package Fermipy v.0.14.1. We selected events with an energy exceeding $800 \mathrm{MeV}$ as a compromise between statistics and good angular resolution. The latter is essential to reduce the effect of the source confusion. We considered Pass 8 data and selected "FRONT + BACK" events (evtype=3) with zenith angles $z \leq z_{\max }=90^{\circ}$, to avoid the Earth limb events and imposed DATA_QUAL $==1$ \&\& LAT_CONFIG $==1$. The considered region of interest (ROI) was a $10^{\circ} \times 10^{\circ}$ square around the cloud center. As a template for the background, the standard galactic background model of the Fermi-LAT collaboration [5] cannot be used since the emission from the molecular clouds is included in the background itself. We generated a customized diffuse interstellar emission model that does not include the emission expected from the selected clouds. For this purpose, we considered the main channels of production of $\gamma$ rays in our selected energy range: the $\pi^{0}$-decay radiation, the inverse Compton scattering and the extragalactic diffuse radiation. We modeled the $\pi^{0}$ emission from the gas map, by considering different surveys, as discussed below. For the inverse Compton component, we used the map ${ }^{\mathrm{S}} \mathrm{Y}^{\mathrm{Z}} 10^{\mathrm{R}} 30^{\mathrm{T}} 150^{\mathrm{C}} 2$ from GALPROP [28]. For the isotropic extragalactic component we derived a model by fitting a $30^{\circ}$ region centered at $b=90^{\circ}$, where the galactic contribution (pion decay and inverse Compton) is minimum. As a starting point, we included the sources from the 3FGL catalog [26] and added, at a latter stage, new sources that appeared to be significant in the TS map where TS stands for Test Statistic defined e.g., in Ref. [29]. The residual maps, obtained after the modeling, are shown in the Appendix. In the likelihood fit, we kept free all diffuse components as well as the normalization of all sources within 3 degrees from the center of the ROI. We derived the spectral energy distributions (SEDs) by fitting each single energy bin with a power law (PL) function of index 2 and a normalization parameter left free to vary. In general, we used the energy bins corresponding to $\Delta \log E=0.125$, except for some cases when the bins have been enlarged to provide adequate statistics of counts.

\section{A. Templates}

We constructed the templates for the clouds and for the background $\pi^{0}$ emission from the radio maps of gas [20,30] or from infrared maps of dust emission [22]. From each map, we cut out the cloud and considered the rest of the gas as background. For nearby clouds, we considered the data from the Planck satellite ${ }^{2}$ which provides a full-sky survey of the dust optical depth. We used the maps of the thermal dust optical depth at $353 \mathrm{GHz}$ [22]. The advantage of using the dust is that it has a linear relation to the interstellar hydrogen, both atomic and molecular hydrogen. Moreover, it is not sensitive to saturation, like $\mathrm{CO}$, and therefore traces also the so-called "dark gas." The Planck data are bidimensional, and can be used only for isolated clouds, for which any other contribution along the line of sight can be neglected.

The DHT clouds instead cannot be considered isolated, thus we need to take into account the background and foreground gas. We used the three-dimensional data cube of $\mathrm{HI}$ from [30], and the cube of $\mathrm{CO}$ from [20] as tracers of molecular hydrogen. For each cloud, we considered the position $\left(l_{0}, b_{0}, v_{0}\right)$, the velocity dispersion $\sigma_{v}$, and the observed extension $\sigma_{r}$ as given in Ref. [19]. We considered a cubic box centered at the center of the cloud, with the width $\Delta v=2 \sqrt{2 \ln 2} \sigma_{v}$ and the side $2 R ; R$ is the radius of the $\mathrm{H}_{2}$ region inferred from $\sigma_{r}$. As explained in Refs. [19,31], because of the different gas density profiles, the radius of the $\mathrm{CO}$ emitting region $\sigma_{r}$ is generally smaller than the radius $R$ of the $\mathrm{H}_{2}$ region. Following Ref. [31], we assumed $R=\eta \sigma_{r}$ with $\eta=1.91$. The remaining $\mathrm{H}_{2}$ is taken as background. We considered HI as background, but not as a signal.

For the Sgr B complex, we followed the methodology of the previous analysis as described in [18] and updated the analysis by using ten years of Pass 8 data. We note that it is impossible to perform a kinetic separation towards the Galactic Center region, and that, due to the high opacity, $\mathrm{CO}$ observations may significantly underestimate the gas density in this region. Therefore we used the Planck opacity map [22] to trace the gas in this region. We adopted a similar data preparation procedure as in other regions, and considered the $0.5^{\circ} \times 0.5^{\circ}$ box to define the Sgr B region as $0.4^{\circ}<l<0.9^{\circ},-0.3^{\circ}<b<0.2^{\circ}$. We used the dust opacity map in this box as our source template and considered the other regions of the map as background.

\section{RESULTS}

\section{A. Spectral energy distribution}

In Fig. 5 we show the SED of the selected GMCs and in Fig. 6 we present the SED of the Sgr B complex. In the same figure, we show the $\gamma$-ray fluxes expected from interactions of the $\mathrm{CR}$ sea with the clouds. For

\footnotetext{
${ }^{2}$ http://www.esa.int/Our_Activities/Space_Science/Planck.
} 


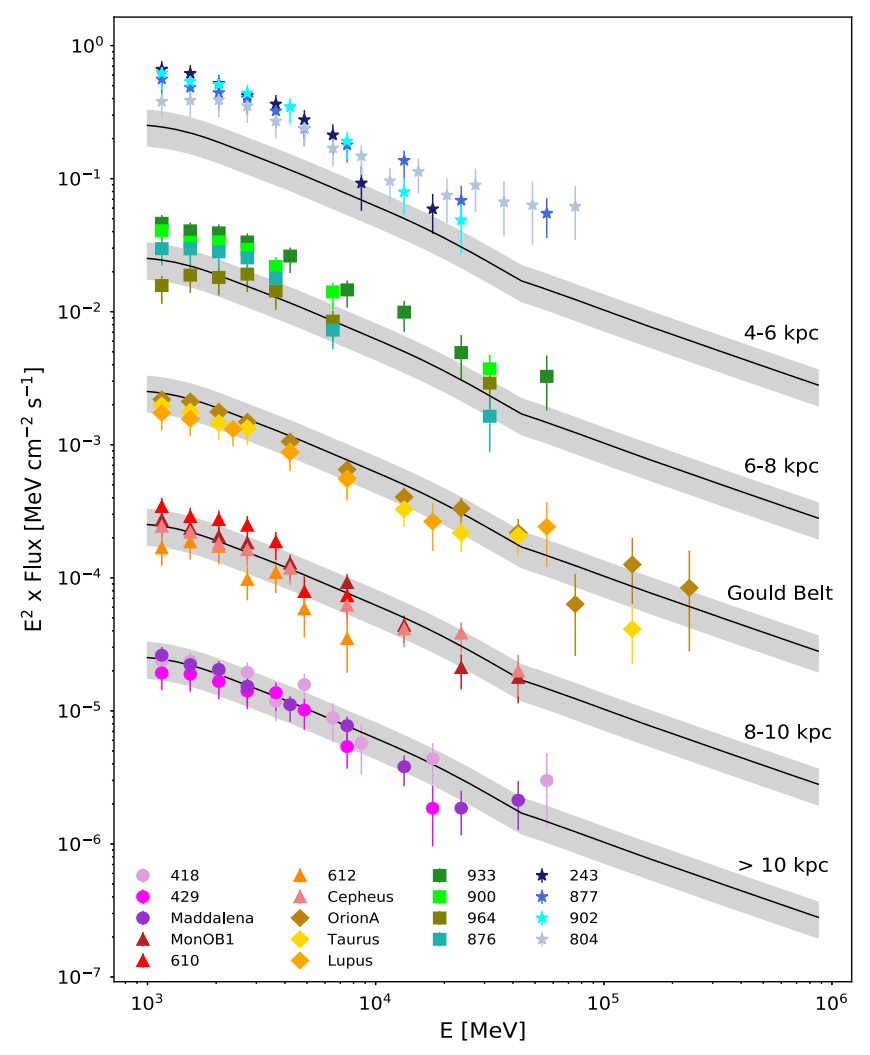

FIG. 5. Spectral energy distributions of GMCs. From the bottom to the top: clouds within the galactocentric distances 10-12 kpc; 8-10 kpc, from the Gould Belt complex, and belong to $6-8 \mathrm{kpc}$ and $4-6 \mathrm{kpc}$ rings. SEDs are normalized to $A=1$. For better visibility, the fluxes corresponding to these zones are separated from each other by scaling them by the factors $10^{\mathrm{n}}$ with $\mathrm{n}=1,2,3$ and 4 . For each set of GMCs, the dashed black lines are the expected SEDs of a cloud with $A=1$ calculated for the local CR proton spectrum reported by the AMS collaboration [12], and using the differential cross-section parametrization from [11]. The parameter that takes into account the contribution of nuclei to the $\gamma$-ray production, $\xi_{N}=1.8$, corresponds to the standard compositions of the interstellar medium and CRs. The shaded gray area indicates the $30 \%$ uncertainty in $\mathrm{M}_{5} / \mathrm{d}_{k p c}^{2}$ which is due to the CO-to- $\mathrm{H}_{2}$ conversion factor.

convenience, when comparing the fluxes from different clouds, the SEDs are normalized to $A=1$. All spectral points, shown in Fig. 5, have a statistical significance $T S>5$. The interactions of the CR sea with the clouds set the minimum level of $\gamma$-ray fluxes from individual clouds. This component of radiation is calculated for the locally measured fluxes of CR protons reported by the AMS collaboration [12]. The dashed gray zones indicate the flux uncertainties introduced by $\approx 30 \%$ uncertainty in the CO-to$\mathrm{H}_{2}$ conversion factor $X_{\mathrm{CO}}$ that affects the value of $A$.

\section{B. Derivation of cosmic ray density}

From the SEDs of the clouds shown in Fig. 5, we can extract the energy density of parent $\mathrm{CR}$ protons. The differential $\gamma$-ray flux given by Eq. (1) can be expressed via the CR density, $\rho_{\mathrm{CR}}=\frac{4 \pi}{c} F\left(E_{p}\right)$ :

$$
F_{\gamma}\left(E_{\gamma}\right) \propto \frac{M_{5}}{d_{k p c}^{2}} \int d E_{p} \frac{d \sigma}{d E_{\gamma}} \frac{4 \pi}{c} \rho_{\mathrm{CR}}
$$

To calculate $\rho_{\mathrm{CR}}$, we used the NAIMA [32] software package. Compared to the average density of interstellar medium, the gas density in GMCs is very high. Therefore, in the energy interval of interest, the $\gamma$-ray production via collisions of CR nuclei with the ambient gas well dominates over other $\gamma$-ray production channels.

The NAIMA package uses a modified presentation of Eq. (4):

$$
\begin{aligned}
F_{\gamma}\left(E_{\gamma}\right) & =\frac{\left\langle n_{H}\right\rangle}{d^{2}} \int d E_{p} \frac{d \sigma}{d E_{\gamma}} \int_{V} d V \frac{4 \pi}{c} \frac{d N_{p}}{d E d V} \\
& =\frac{\left\langle n_{H}\right\rangle}{d^{2}} \int d E_{p} \frac{d \sigma}{d E_{\gamma}} \frac{4 \pi}{c} \frac{d N_{p}}{d E} .
\end{aligned}
$$

To avoid large uncertainties, we considered only the $\gamma$-ray fluxes above $\sim 1 \mathrm{GeV}$ which trace $\gtrsim 10 \mathrm{GeV}$ CR protons. We assume a simple power law as the initial form of the CR distribution, $d N_{p} / d E=F_{0}\left(E / E_{0}\right)^{-\alpha}$, and derive the parameters $F_{0}$ and $\alpha$ from the distributions of $\gamma$ rays and

TABLE III. The spectral indices and CR proton densities at $10 \mathrm{GeV}$ derived from the $\gamma$-ray and $\mathrm{CO}$ data at the locations of clouds as indicated in Fig. 3. Errors on the normalization result from the sum in quadrature of the statistical error deriving from the fit and the $30 \%$ uncertainty on the $A$ parameter.

\begin{tabular}{lccc}
\hline \hline Cloud & $R_{\text {gal }}[\mathrm{kpc}] \rho_{\mathbf{0}, \mathbf{C R}}\left[10^{-12} \mathrm{GeV}^{-1} \mathrm{~cm}^{-3}\right]$ & $\alpha$ \\
\hline 418 & 10.6 & $1.7 \pm 0.5$ & $2.69 \pm 0.05$ \\
429 & 10.3 & $1.4 \pm 0.4$ & $2.74 \pm 0.05$ \\
Maddalena & 10.1 & $1.8 \pm 0.6$ & $2.93 \pm 0.06$ \\
MonOB1 & 9.1 & $1.9 \pm 0.6$ & $2.99 \pm 0.06$ \\
610 & 8.9 & $2.3 \pm 1.1$ & $2.79 \pm 0.04$ \\
612 & 8.7 & $1.3 \pm 0.4$ & $2.80 \pm 0.09$ \\
Cepheus & 8.6 & $1.7 \pm 0.5$ & $2.87 \pm 0.07$ \\
OrionA & 8.4 & $1.5 \pm 0.5$ & $2.83 \pm 0.05$ \\
Taurus & 8.4 & $1.4 \pm 0.5$ & $2.89 \pm 0.05$ \\
Lupus & 8.2 & $1.1 \pm 0.4$ & $2.74 \pm 0.10$ \\
933 & 7.1 & $3.2 \pm 1.1$ & $2.69 \pm 0.02$ \\
900 & 6.6 & $2.7 \pm 0.8$ & $2.74 \pm 0.03$ \\
964 & 6.4 & $1.3 \pm 0.4$ & $2.56 \pm 0.04$ \\
876 & 6.0 & $2.3 \pm 0.7$ & $2.82 \pm 0.03$ \\
243 & 5.8 & $4.8 \pm 1.4$ & $2.86 \pm 0.03$ \\
877 & 5.5 & $3.9 \pm 1.2$ & $2.69 \pm 0.02$ \\
902 & 5.4 & $4.4 \pm 1.3$ & $2.74 \pm 0.02$ \\
804 & 4.6 & $3.0 \pm 0.9$ & $2.61 \pm 0.02$ \\
Sgr B & 0.1 & $0.98 \pm 0.06$ & $2.80 \pm 0.03$ \\
AMS02 & & 1.12 & $2.8^{\mathrm{a}}$ \\
\hline \hline
\end{tabular}

${ }^{\mathrm{a}}$ From fitting of experimental points on the energy range 20-200 GeV. 
the ambient gas. Initially we set $\left\langle n_{H}\right\rangle=1 \mathrm{~cm}^{-3}$ and $d_{k p c}=1$, so that the normalization is $F_{0}^{\prime}=\frac{\left\langle n_{H}\right\rangle}{d_{\mathrm{kpc}}^{2}} F_{0}$. Then, the normalized CR density is linked to $F_{0}^{\prime}$ through the parameter A:

$$
\rho_{0, \mathrm{CR}}=\frac{F_{0}}{V}=\frac{m_{p}}{10^{5} M_{\odot}} A^{-1} F_{0}^{\prime} .
$$

In this way, the systematic errors in the calculations of $F_{0}$ are reduced to the uncertainty of the parameter $A=$ $M_{5} / d_{\mathrm{kpc}}^{2}$ caused basically by the uncertainty related to the conversion factor $X_{\mathrm{CO}}$. For all clouds, the derived values of $\rho_{0}$ and $\alpha$ are listed in Table III. In Fig. 7, we show these values as a function of the galactocentric distance, $R_{\text {gal }}$. We compared the derived values to the local values for cosmic proton as measured by AMS02. Since AMS02 data are not well represented by a single power law distribution to guarantee a fair comparison we fitted the data in restricted energy intervals. In the interval of our interest i.e., 20-200 GeV, the best power law index resulted to be $\alpha=2.8$. Note that, Taurus and Lupus have different spectral indices because the spectrum of Taurus extends to higher energies, and therefore it is less influenced by the flattening of the spectrum at energies of a few $\mathrm{GeV}$.

\section{DISCUSSION}

The results of the previous section allow a robust conclusion for all the clouds with galactocentric distances larger than $8 \mathrm{kpc}$, independently of their location in the Galaxy. The CR density in these objects is close to the locally measured CR flux reported by the AMS collaboration. Figure 5 shows a good agreement between the predicted and observed $\gamma$-ray fluxes from three regions representing (i) the Gould Belt complex, (ii) the $8-10 \mathrm{kpc}$ ring and (iii) the periphery beyond $\geq 10 \mathrm{kpc}$. While the data relevant to the Gould Belt clouds could be interpreted as a result of the dominant contribution by local accelerators to the measured CR flux, the $\gamma$-ray data from other GMCs, in particular, Maddalena and clouds \#418 and $\# 429$, which lie further away $(\gtrsim 1 \mathrm{kpc})$, exclude this scenario. Besides, the same nominal flux of $\gamma$ rays calculated for the AMS-measured CR flux is observed from cloud \#964 located in the inner Galaxy, at a distance of $6 \mathrm{kpc}$ from the GC. The constancy of the derived densities and the spectral indices of CRs tell us that, most likely, we deal with the sea of CRs.

Remarkably, the same level of CR density has been found by Yang et al. [18] also for a quite different part of the Galaxy, in the Galactic Center region. The analysis of $\gamma$ rays from the Sgr B complex conducted in this work with an almost doubled photon statistics, confirms the conclusions of Ref. [18] about the low flux of CRs in the CMZ. The $\gamma$-ray SED from this region is shown in Fig. 6. Below $10 \mathrm{GeV}$, we can see a very good agreement with the

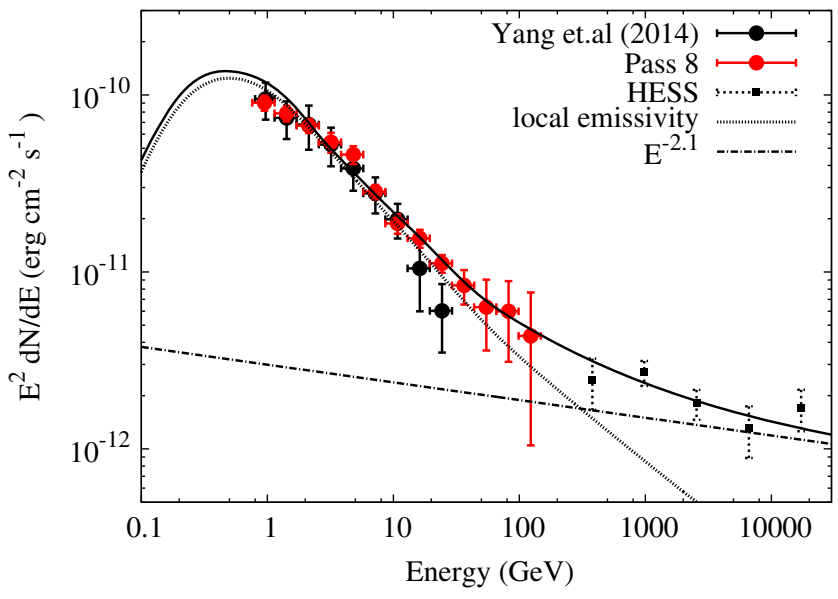

FIG. 6. $\quad \gamma$-ray fluxes from the Sgr B complex. The red circles are from the ten-years Pass 8 data derived in this work, the black circles are from Ref. [18]. The black squares represent the diffuse $\gamma$-ray flux reported by the H.E.S.S. collaboration [33]. The dotted line is the predicted gamma-ray spectrum under the assumption that the CR flux in the Sgr B region is the same as the local CR spectrum measured by AMS02. The dot-dashed line is a powerlaw component with the photon index of -2.1. In superposition with the component linked to the CR sea (solid curve), it explains the hardening of the $\gamma$-ray spectrum above $10 \mathrm{GeV}$ and almost entirely the flux at $\mathrm{TeV}$ energies.

previously reported fluxes [18]. Above $10 \mathrm{GeV}$, the new analysis reveals a noticeable hardening which is naturally explained by the presence of the diffuse component of very high-energy $\gamma$ rays discovered from the same region with the H.E.S.S. telescopes [33]. Because of the harder spectrum, the contribution of this component below $10 \mathrm{GeV}$ becomes less than $10 \%$. The parent particle population responsible for this component is provided, presumably, by the PeVatron(s) located within the central $10 \mathrm{pc}$ region of the GC [34]. Meanwhile, the low-energy component is perfectly explained by the sea of galactic CRs. To a certain extent, this is an unexpected result, given the presence of several potentially powerful CR accelerators linked to the central supermassive black hole and the high starburst rate in this area. A plausible explanation of this result could be the effective escape of low-energy CRs from the inner parts of the GC, e.g., due to the fast convection before they could propagate to large distances and approach the Sgr B complex.

Gaggero et al. [36] proposed an alternative interpretation of the hard-spectrum multi-TeV CR component revealed from the H.E.S.S. observations of the CMZ. They argued that the enhanced CR flux towards the GC could be explained by a specific CR transport model assuming a position-dependent diffusion coefficient. However, this model cannot address the $1 / r$ distribution of CR density above $10 \mathrm{TeV}$ as derived from the H.E.S.S. observations within the CMZ; the authors admit [36] the need of existence of additional CR source(s) at the heart of GC. Moreover, the very low density of $\leq 1 \mathrm{TeV}$ CRs derived 
from Fermi-LAT $\gamma$-ray observations at the position of Sgr B complex (see Fig. 6) excludes the interpretation of Ref. [36].

At the same time, we found significant deviations from the homogeneous CR sea at locations of some GMCs in the inner Galaxy, at galactocentric distances between 4 and $8 \mathrm{kpc}$. Some clouds show enhanced, by a factor of 2 to 5 , $\gamma$-ray fluxes, and somewhat harder energy spectra compared to $\gamma$ rays induced by the sea of CRs (see Fig. 5). A tendency of increase of the density of CRs had been reported earlier based on the analysis of the diffuse $\gamma$ radiation of the GD [5,6,37-39]. In Fig. 7 we show the CR density at energy for proton energies greater than $10 \mathrm{GeV}$ derived from the diffuse gamma-ray measurements [5] and from individual clouds (this work), as a function of the galactocentric radius. In the same figure we show also the theoretical curve from Ref. [35]. The latter has been calculated under the assumption of spatial anticorrelation of the diffusion coefficient with the source distribution, to explain the sharp maximum of the CR density at $\approx 2-5 \mathrm{kpc}$ as reported by Ref. [5]. While the method based on the diffuse $\gamma$-ray emission gives the average density of CRs inside the galactocentric rings, $\gamma$ rays from individual clouds provide direct information about $\mathrm{CR}$ density in specific regions localized within a few tens of parsecs. This is important information which can tell us whether the enhanced $\gamma$-ray emission of these clouds is a result of (1) the global variation of the level of the CR sea or (2) it is caused by an additional component of relativistic particles on top of the CR sea. The CR densities presented in Fig. 7 show fluctuations in the 4 to $8 \mathrm{kpc}$ region. The effect is well

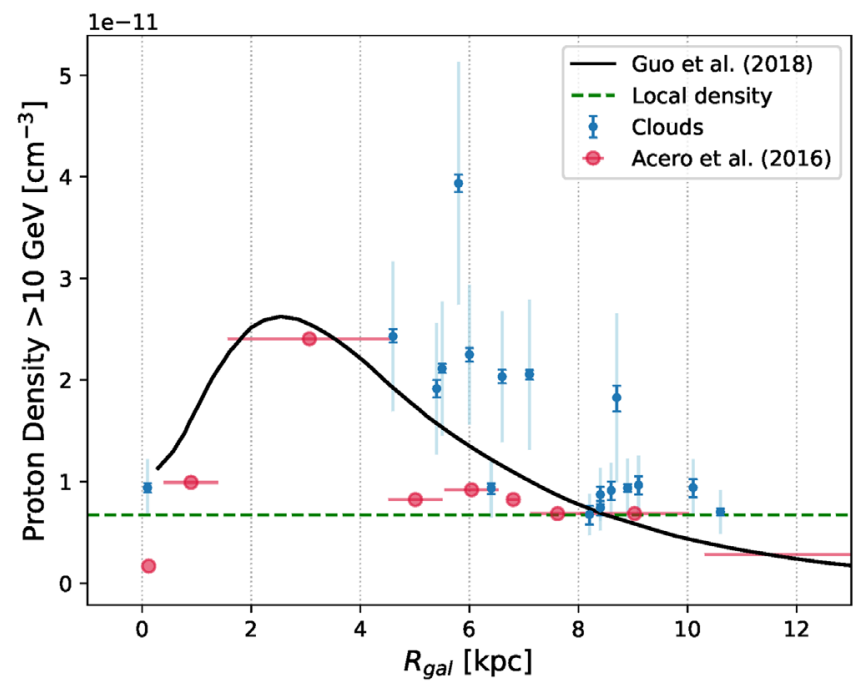

FIG. 7. Energy density of protons derived above $10 \mathrm{GeV}$. For each cloud are indicated the statistical (thick lines) and the systematic (thin lines) error bars. The red points are taken from Ref. [5]. The black curve indicates the model of [35], while the green dashed line indicates the local proton density derived from the measurements of Ref. [12]. visible especially for three clouds, \#964, \#876 and \#243, located at similar, $R \simeq 6 \mathrm{kpc}$ galactocentric distances (see also Fig. 5), when considering only the statistical uncertainties. Note that the density derived for cloud \#964 is rather close to the density derived from the analysis of the diffuse $\gamma$-ray emission; the density in clouds \#876 and \#243 exceeds that level by a factor of 2 to 4 .

This could be a direct consequence of the fact that most of the active star-forming regions, and therefore, the potential particle accelerators, are located within the 4-6 kpc ring. The dimension of regions with enhanced $\mathrm{CR}$ density depends on the strength and the age of the accelerator. For SNRs with $10^{51}$ erg mechanical energy release, the CR density in the surrounding ISM can exceed the level of the CR sea up to $100 \mathrm{pc}$ from the accelerator [40]. In the case of young stellar clusters of age $\geq 10^{6}$ years with mechanical luminosity of stellar winds of $10^{38-39} \mathrm{erg} / \mathrm{s}$, the regions with enhanced CR density can extend well beyond $100 \mathrm{pc}$ [41]. Since both types of accelerators are tightly linked to the star-forming regions, they are often surrounded by GMCs. The presence of dense gaseous regions close to particle accelerators creates favorable conditions for effective production of $\gamma$ rays [40]. High-energy $\gamma$ rays from massive molecular clouds provide direct information about the density of CRs in different locations of the Milky Way. The results of this work prove the feasibility of the method and demonstrate that $\gamma$ rays from GMCs can serve as unique CR barometers. An indepth study of this issue requires significantly larger statistics of gamma-ray emitting clouds and denser coverage of galactocentric distances. Unfortunately, in the context of this task, the potential of Fermi-LAT is almost saturated. Even the doubling of $\gamma$-ray photon statistics, after another ten years of operation of the telescope, cannot significantly increase the number of detectable gamma-ray emitting GMCs. For a breakthrough, a new generation gamma-ray telescope is needed with a sensitivity improved compared to Fermi-LAT, by a factor of 3 or more.

\section{ACKNOWLEDGMENTS}

The authors would like to thank Jim Hinton and Luigi Tibaldo for the fruitful discussions and Lucia Ambrogi for her help on the modeling of nonthermal emission. S.C. acknowledges the National Science Centre, Poland Grant No. DEC-2017/27/B/ST9/02272.

\section{APPENDIX A: MASS DETERMINATION}

To have a consistent estimation of the mass, we derived it from our cut-out template, as the mass given in the catalog by [19] might refer to a slightly different shape. Following Rosolowsky [31], the mass and consequently the parameter $A=M_{5} / \mathrm{d}_{\mathrm{kpc}}^{2}$ is computed from the column density, derived from the $\mathrm{CO}$ emission: 


$$
M=2 m_{p} X_{\mathrm{CO}} \sum_{i, j} W_{i j}\left(l_{i}, b_{i}\right) \Delta l \Delta b\left(\frac{\pi}{180}\right)^{2} d^{2},
$$

where $W_{i j}\left(l_{i}, b_{i}\right) \equiv \int_{v_{\min }}^{v_{\max }} T_{\mathrm{CO}}(l, b, v) d v$ is the integrated brightness temperature of $\mathrm{CO}$ for every pixel. We can notice that the mass is directly related to the distance $d^{2}$, since it links the physical extension to the observed angular one. This allows us to have an estimation of the $A$ factor directly from the $\mathrm{CO}$ data, which does not depend directly on the estimation of the distance and the mass:

$$
A=\frac{M_{5}}{d_{\mathrm{kpc}}^{2}} \approx 32 \sum_{i, j} W_{i j}\left(l_{i}, b_{i}\right) \Delta l \Delta b\left(\frac{\pi}{180}\right)^{2} .
$$

As a consequence the uncertainties on the mass and on the distance cancel out and the only uncertainty on $A$ comes from the factor $X_{\mathrm{CO}}$, which is considered to be of $30 \%$ as suggested by Bolatto et al.[42].

The helium fraction of the ISM here is not taken into account in the calculation of the mass, differently from what is done in [19] where the authors include it as a further factor 1.36. Helium contribution in $\gamma$-ray emission from $\mathrm{pp}$ interaction is already accounted in the nuclear enhancement factor, which we assumed to be 1.8 as in [11].

\section{APPENDIX B: GAS DISTRIBUTION}

Differently from its atomic counterpart (HI), the molecular hydrogen $\left(\mathrm{H}_{2}\right)$ is not uniformly distributed in the GD. The molecular gas in the interstellar medium (ISM) rather tends to concentrate in dense massive clouds. Correspondingly, the column density of the gas in a given direction is composed of contributions from most massive clouds.

The best tracer of molecular hydrogen in optically thin regions is the mm emission of ${ }^{12} \mathrm{CO}(1 \rightarrow 0)$. The column density in the given direction is determined as

$$
n(l, b)\left[\mathrm{cm}^{-2}\right]=X_{\mathrm{CO}} \int \mathrm{d} v T_{b}(l, b, v)\left[\mathrm{km} \mathrm{s}^{-1} \mathrm{~K}\right],
$$

where, following [42], the CO-to- $\mathrm{H}_{2}$ conversion factor, $X_{\mathrm{CO}}$, is set to $X_{\mathrm{CO}}=2 \times 10^{20} \mathrm{~cm}^{-2} \mathrm{~K}^{-1} \mathrm{~km}^{-1} \mathrm{~s}$. In this paper, we use the $\mathrm{CO}$ data from [20].

In the analysis, the atomic hydrogen is considered only as background gas since it is difficult to isolate the $\mathrm{HI}$ in the cloud from the rest of the column density. For the atomic counterpart we considered the $21-\mathrm{cm}$ emission line data cube from the HI4PI collaboration [30]. As for the molecular gas, the HI density is directly proportional to the brightness temperature of the $21-\mathrm{cm}$ line. In this case, for the conversion factor we use the value from [30]: $X_{\mathrm{HI}}=1.83 \times 10^{18} \mathrm{~cm}^{-2} \mathrm{~K}^{-1} \mathrm{~km}^{-1} \mathrm{~s}$. This conversion factor is derived from the assumption of optically thin emission and must be corrected for possible absorption caused by cooler components. Following for example [43], the corrected column density is calculated as

$$
\begin{aligned}
& n_{\mathrm{HI}}(l, b)\left[\mathrm{cm}^{-2}\right] \\
& \quad=-X_{\mathrm{HI}} T_{s} \int d v \ln \left(1-\frac{T_{b}}{T_{s}-T_{0}}\right)\left[\mathrm{K} \mathrm{km} \mathrm{s}^{-1}\right],
\end{aligned}
$$

where $T_{s}$ is the average spin temperature of the interstellar Hi [44]. $T_{s}$ is an effective parameter that describes the mixture of warm and cold neutral components:

$$
T_{s}=T_{C N M} \frac{n_{w}+n_{c}}{n_{c}} .
$$

The value of $T_{s}$ can be measured in the presence of strong continuum sources in the background as described, for example, in [45]. These measurements are available only for specific regions of the Milky Way, for other locations we need to rely on an educated guess. For our case we considered that the maximum measured brightness temperature in these clouds is $143 \mathrm{~K}$, so we assumed, as lower limit, $T_{s, \min }=150 \mathrm{~K}$; as an upper limit we assumed $T_{s, \max }=500 \mathrm{~K}$, that results from Eq. (B3) by taking $n_{w}=$ $90 \%$ of warm neutral medium and $T_{C N M}=50 \mathrm{~K}$ [46].

In Fig. 8 we show the derived $\mathrm{H}_{2}$ and $\mathrm{HI}$ distributions by integrating $T_{b}(l, b, v)$ in the regions that coincide with the cloud spatial templates, defined as explained in the main text. The pink area represents the cloud extension along the line of sight.

\section{Systematic uncertainties}

We considered the following sources of systematic errors for the spectral points.

a. New sources. - At the moment of writing the 4FGL catalog has not been publicly released, so we made use of the 3FGL catalog of $\gamma$-ray sources. The 3FGL catalog is based on four years of data, so we expect to reveal several new sources. In few cases some sources appeared at the edges of our analyzed clouds. We evaluated the uncertainties deriving from the new sources with this procedure: we first fitted the data with a model that included (besides the galactic and extragalactic emission) only the known point sources from the 3FGL catalog and derived the SED for the cloud with this model. Then we identified as new sources all the significant $(\mathrm{TS}>20)$ residuals in the R.O.I. and included them in the model. We refitted until the TS map was clear from significant sources. We checked also the residual maps to be sure that they were ranging from -3 to 3 sigma (Fig. 9). With this new model we derived a new SED for the clouds. The difference between the two SEDs was then considered as an additional uncertainty. The difference from the two sets of spectral points is estimated to be at worst $\sim 20 \%$ for what concerns the normalization and does not affect the slope. This uncertainty is included in the systematic errors for each cloud.

b. Radial dependent CR distribution.-Studies of the diffuse $\gamma$-ray emission showed that the cosmic ray spectra have different shapes at different distances from the GC. 


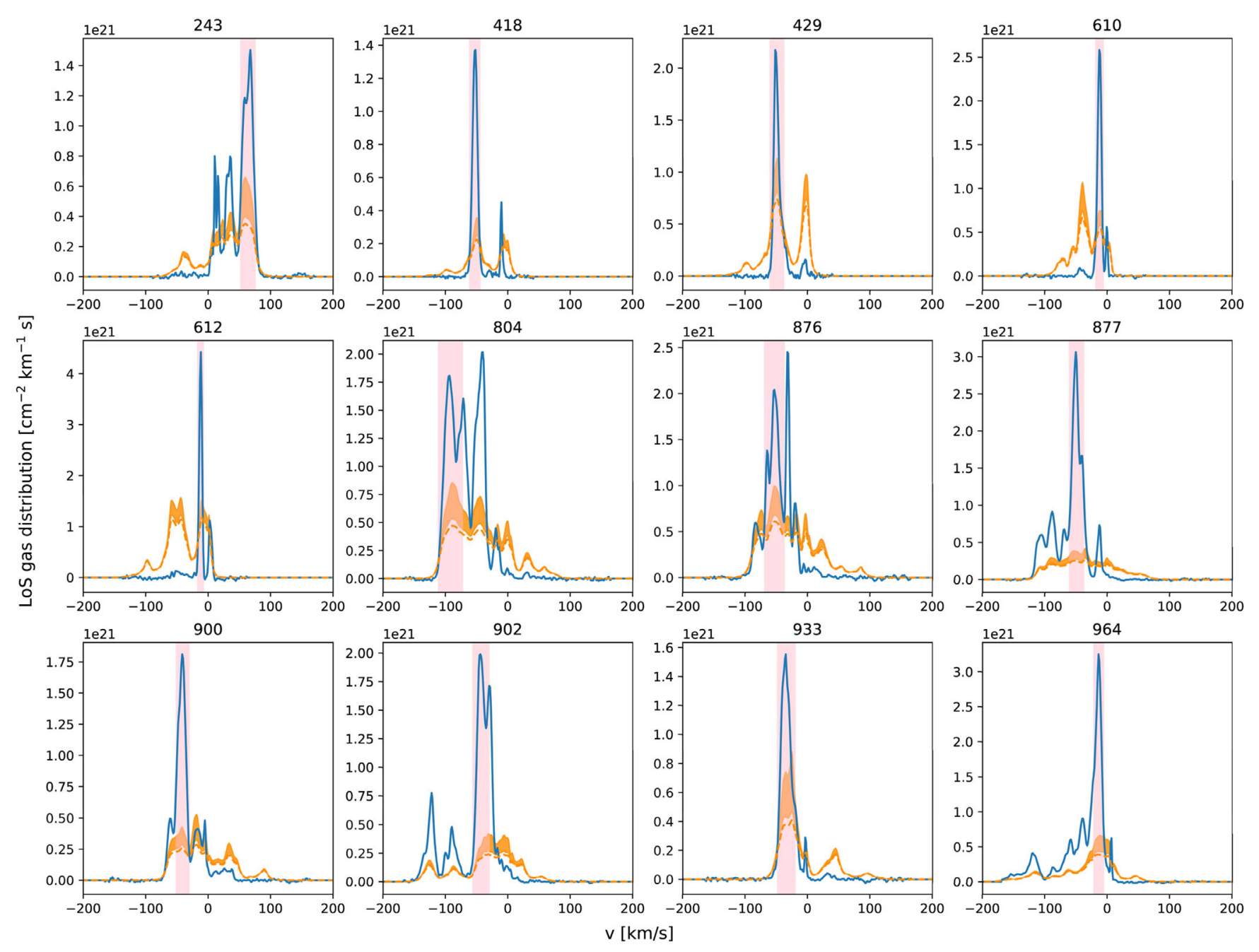

FIG. 8. Gas distribution along the line of sight in the regions of the analyzed MCs. The pink area delimits the velocity-range of the cloud. Blue lines represent the distribution of molecular hydrogen for which we assumed $\mathrm{X}_{\mathrm{CO}}=2 \times 10^{20} \mathrm{~cm}^{-2} \mathrm{~K}^{-1} \mathrm{~km}^{-1} \mathrm{~s}$. The orange dashed lines trace the HI distribution, assuming optically thin emission with a conversion factor of $\mathrm{X}_{\mathrm{HI}}=1.8 \times 10^{18} \mathrm{~cm}^{-2} \mathrm{~K}^{-1} \mathrm{~km}^{-1} \mathrm{~s}$. The orange area takes into account the variation of density while assuming a spin temperature correction with $150 \mathrm{~K} \leq T_{s} \leq 500 \mathrm{~K}$.

In particular [5,6] show that the CR density increases and spectrum hardens towards the inner Galaxy. In our analysis we assumed the CR radial distribution to be uniform in the considered ROI. We evaluated the effect of the radial dependence of the background [6] by performing an equivalent analysis but assuming a radial dependent background model. We divided the gas in six galactocentric rings and assigned to each of them the corresponding normalization and spectral index of CRs derived in [6]. The difference between the SEDs derived with the two distinct backgrounds is lower than $10 \%$ in terms of normalization.

c. Optically thin HI.-As discussed before, the map of HI from the HI4PI collaboration does not have any assumption on the optical thickness of the gas. We cannot assume that the HI, especially in the GD, is optically thin as this could lead to an underestimation of the background. We corrected the computed column density by using Eq. (B2) with different values of $T_{s}$ and tested the influence of this variation by performing an independent analysis on the same data. Even if for all selected clouds the contribution of the molecular gas is dominant over the atomic, in terms of column density, the resulting spectra seem to be affected by the choice of the Hi background by a factor that could vary from $20 \%$ to $30 \%$.

All these contributions were then summed in quadrature to obtain the final systematic uncertainty.

\section{Counts profile}

In order to check the results of our likelihood fit, we compared the integrated profile of counts as a function of the right ascension (RA) with the model resulting after the fit, see Fig. 10. The profiles were obtained by integrating over the declination (DEC) in a slice that corresponded to the cloud extension. In the plot we show the fitted total model, together with the model of 

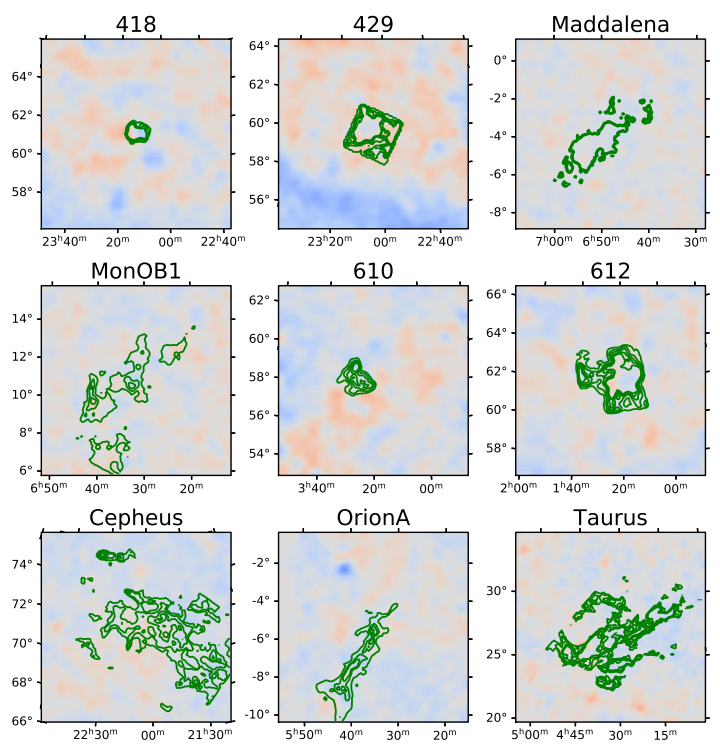

Taurus

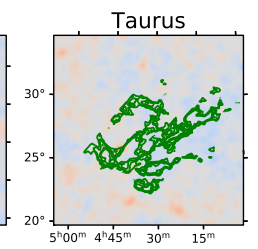

933
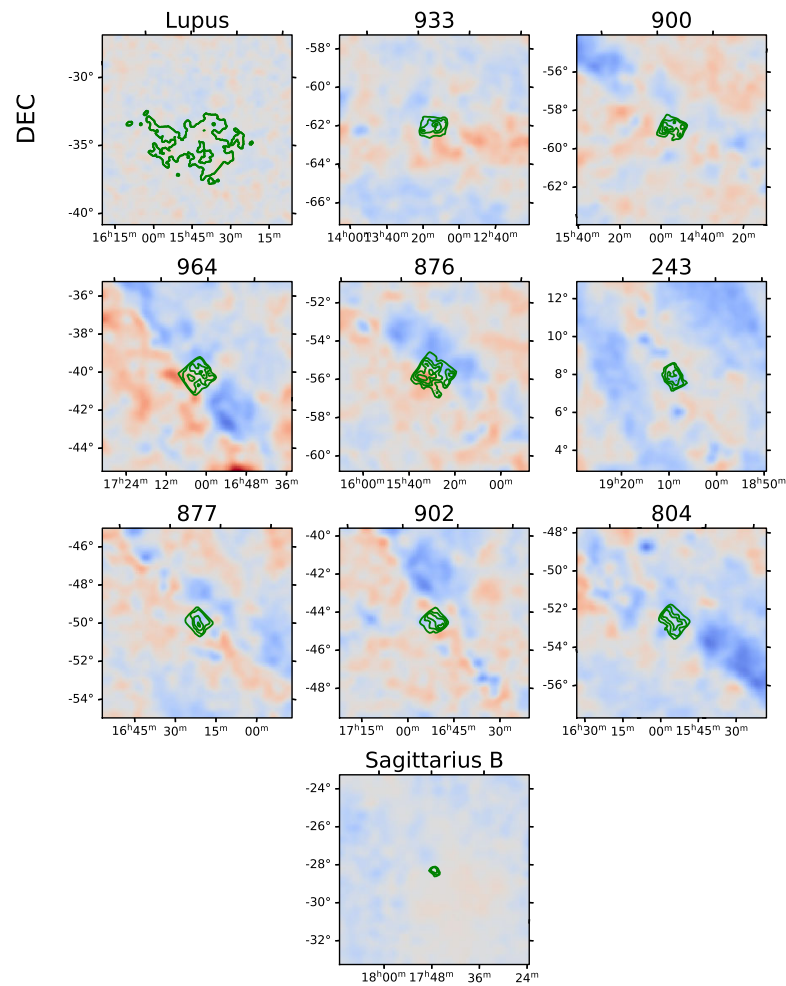

RA

FIG. 9. Residual maps of the analyzed clouds. The green contours show the shapes of clouds as seen from the CO map, for DHT clouds, and from the dust maps of nearby clouds.

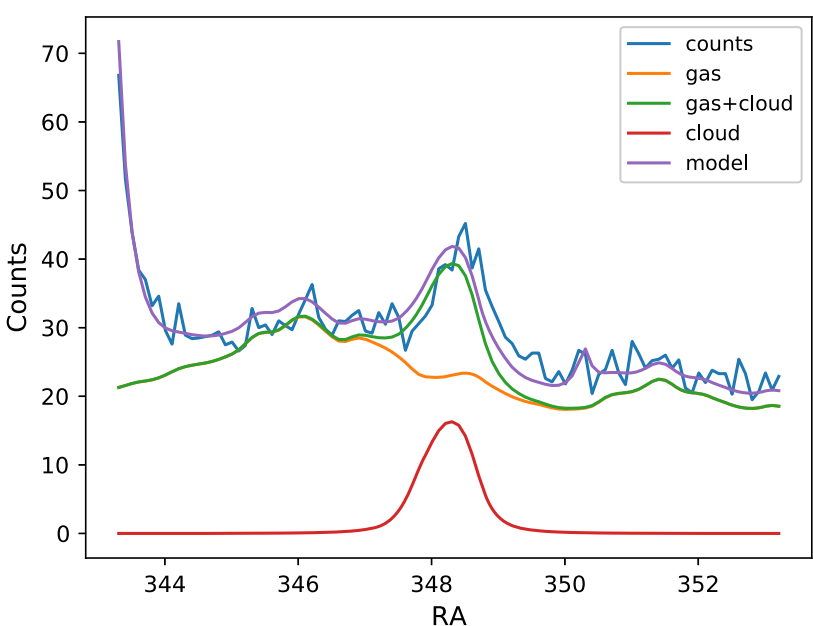

(a)

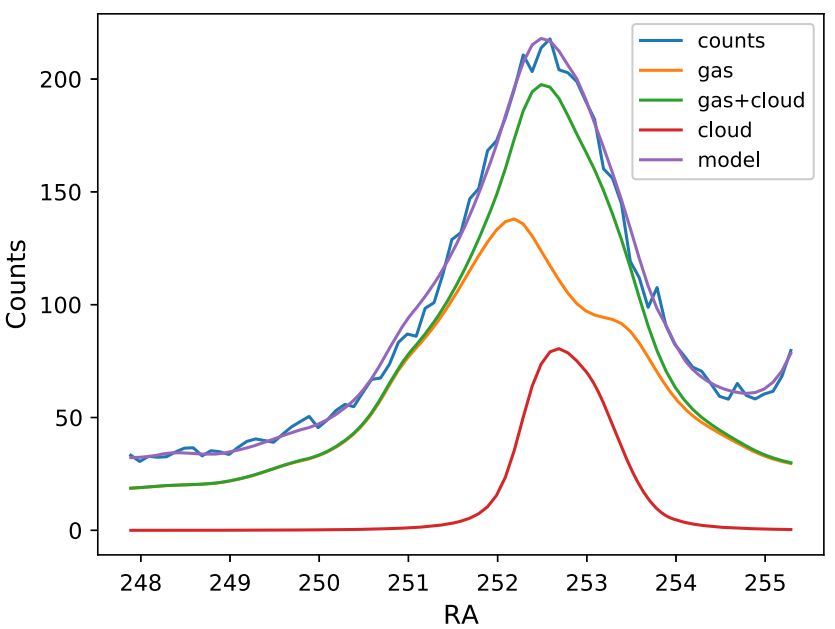

(b)

FIG. 10. Comparison between the data counts and the model counts of the cloud and of the background gas, derived in the analyzed energy range ( $>800 \mathrm{MeV})$ after the fit, from a slice in DEC correspondent to the extension of the cloud. We show two examples: in (a) cloud 418 that is in the outer Galaxy, in (b) cloud 902, located in the inner Galaxy.

the cloud, the background gas and the sum of the two. We can see that the total model well represents the observed counts and that the pion decay emission of the gas provides, as expected, the dominant contribution to the observed emission. 
[1] F. W. Stecker, Astrophys. J. 212, 60 (1977).

[2] F. A. Aharonian and A. M. Atoyan, Astron. Astrophys. 362, 937 (2000), https://ui.adsabs.harvard.edu/abs/2000A\%26A. ..362..937A/abstract.

[3] A. W. Strong, I. V. Moskalenko, and O. Reimer, Astrophys. J. 613, 962 (2004).

[4] A. Strong and J. Mattox, Astron. Astrophys. 308, L21 (1996), https://ui.adsabs.harvard.edu/abs/1996A\%26A.. .308L..21S/abstract.

[5] F. Acero (Fermi-LAT Collaboration), Astrophys. J. Suppl. Ser. 223, 26 (2016).

[6] R. Yang, F. Aharonian, and C. Evoli, Phys. Rev. D 93, 123007 (2016).

[7] F. Aharonian, Space Sci. Rev. 99, 187 (2001).

[8] S. Casanova, F. A. Aharonian, Y. Fukui, S. Gabici, D. I. Jones, A. Kawamura, T. Onishi, G. Rowell, H. Sano, K. Torii et al., Publ. Astron. Soc. Jpn. 62, 769 (2010).

[9] C. J. Cesarsky and H. J. Volk, Astron. Astrophys. 70, 367 (1978), http://adsabs.harvard.edu/full/1978A\%26A... $.70 . .367 \mathrm{C}$.

[10] S. Gabici, F. A. Aharonian, and P. Blasi, Astrophys. Space Sci. 309, 365 (2007).

[11] E. Kafexhiu, F. Aharonian, A. M. Taylor, and G. S. Vila, Phys. Rev. D 90, 123014 (2014).

[12] M. Aguilar et al. (AMS Collaboration), Phys. Rev. Lett. 114, 171103 (2015).

[13] S. W. Digel, I. Grenier, A. Heithausen, S. Hunter, and P. Thaddeus, Astrophys. J. 463, 609 (1996).

[14] A. Neronov, D. Malyshev, and D. V. Semikoz, Astron. Astrophys. 606, A22 (2017).

[15] R.-z. Yang, E. de Oña Wilhelmi, and F. Aharonian, Astron. Astrophys. 566, A142 (2014).

[16] A. Abdo, M. Ackermann, M. Ajello, L. Baldini, J. Ballet, G. Barbiellini, D. Bastieri, B. Baughman, K. Bechtol, R. Bellazzini et al., Astrophys. J. 710, 133 (2010).

[17] M. Ackermann, M. Ajello, A. Allafort, L. Baldini, J. Ballet, G. Barbiellini, D. Bastieri, K. Bechtol, R. Bellazzini, B. Berenji et al., Astrophys. J. 755, 22 (2012).

[18] R.-z. Yang, D. I. Jones, and F. Aharonian, Astron. Astrophys. 580, A90 (2015).

[19] T. S. Rice, A. A. Goodman, E. A. Bergin, C. Beaumont, and T. M. Dame, Astrophys. J. 822, 52 (2016).

[20] T. M. Dame, D. Hartmann, and P. Thaddeus, Astrophys. J. 547, 792 (2001).

[21] C. Zucker, J. S. Speagle, E. F. Schlafly, G. M. Green, D. P. Finkbeiner, A. A. Goodman, and J. Alves, Astrophys. J. 879, 125 (2019).

[22] P. A. Ade, N. Aghanim, M. Arnaud, M. Ashdown, J. Aumont, C. Baccigalupi, A. Balbi, A. Banday, R. Barreiro, J. Bartlett et al., Astron. Astrophys. 536, A19 (2011).

[23] M. Reid, K. Menten, X. Zheng, A. Brunthaler, and Y. Xu, Astrophys. J. 705, 1548 (2009).
[24] E. Schlafly, G. Green, D. Finkbeiner, H.-W. Rix, E. Bell, W. Burgett, K. Chambers, P. Draper, K. Hodapp, N. Kaiser et al., Astrophys. J. 786, 29 (2014).

[25] F. Comerón, in Handbook of Star Forming Regions (Astronomical Society of the Pacific, 2008), Vol. 2, p. 295.

[26] F. Acero (Fermi-LAT Collaboration), Astrophys. J. Suppl. Ser. 218, 23 (2015).

[27] H. Abdalla et al., Astron. Astrophys. 612, A1 (2018).

[28] A. E. Vladimirov, S. W. Digel, G. Jóhannesson, P. F. Michelson, I. V. Moskalenko, P. L. Nolan, E. Orlando, T. A. Porter, and A. W. Strong, Comput. Phys. Commun. 182, 1156 (2011).

[29] J. R. Mattox, D. Bertsch, J. Chiang, B. Dingus, S. Digel, J. Esposito, J. Fierro, R. Hartman, S. Hunter, G. Kanbach et al., Astrophys. J. 461, 396 (1996).

[30] N. B. Bekhti, L. Flöer, R. Keller, J. Kerp, D. Lenz, B. Winkel, J. Bailin, M. Calabretta, L. Dedes, H. Ford et al., Astron. Astrophys. 594, A116 (2016).

[31] E. Rosolowsky, J. Pineda, J. Kauffmann, and A. Goodman, Astrophys. J. 679, 1338 (2008).

[32] V. Zabalza, Proc. Sci., ICRC2015 (2015) 922, https://pos .sissa.it/236/922/pdf.

[33] F. Aharonian et al., Nature (London) 439, 695 (2006).

[34] A. Abramowski, F. Aharonian, F. A. Benkhali, A. Akhperjanian, E. Angüner, M. Backes, A. Balzer, Y. Becherini, J. B. Tjus, D. Berge et al., Nature (London) 531, 476 (2016).

[35] Y.-Q. Guo and Q. Yuan, Phys. Rev. D 97, 063008 (2018).

[36] D. Gaggero, D. Grasso, A. Marinelli, M. Taoso, and A. Urbano, Phys. Rev. Lett. 119, 031101 (2017).

[37] F. W. Stecker, Phys. Rev. Lett. 35, 188 (1975).

[38] J. Bloemen, A. Strong, H. Mayer-Hasselwander, L. Blitz, R. Cohen, T. Dame, D. Grabelsky, P. Thaddeus, W. Hermsen, and F. Lebrun, Astron. Astrophys. 154, 25 (1986), http:// adsabs.harvard.edu/full/1986A\%26A...154...25B.

[39] M. Pothast, D. Gaggero, E. Storm, and C. Weniger, J. Cosmol. Astropart. Phys. 10 (2018) 045.

[40] F. A. Aharonian and A. M. Atoyan, Astron. Astrophys. 309, 917 (1996), http://adsabs.harvard.edu/full/1996A\%26A.. .309..917A.

[41] F. Aharonian, R. Yang, and E. de Oña Wilhelmi, Nat. Astron. 3, 561 (2019).

[42] A. D. Bolatto, M. Wolfire, and A. K. Leroy, Annu. Rev. Astron. Astrophys. 51, 207 (2013).

[43] Y. Sofue, Mon. Not. R. Astron. Soc. 468, 4030 (2017).

[44] J. M. Dickey, S. Strasser, B. Gaensler, M. Haverkorn, D. Kavars, N. McClure-Griffiths, J. Stil, and A. Taylor, Astrophys. J. 693, 1250 (2009).

[45] A. J. Walsh, H. Beuther, S. Bihr, K. G. Johnston, J. R. Dawson, J. Ott, S. N. Longmore, Q. N. Luong, R. S. Klessen, S. Ragan et al., Mon. Not. R. Astron. Soc. 455, 3494 (2016).

[46] C. Heiles and T. Troland, Astrophys. J. 586, 1067 (2003). 